\title{
BOLD cardiovascular magnetic resonance at 3.0 tesla in myocardial ischemia
}

Robert Manka ${ }^{1}$, Ingo Paetsch ${ }^{1,2}$, Bernhard Schnackenburg ${ }^{3}$, Rolf Gebker ${ }^{1}$, Eckart Fleck ${ }^{1}$, Cosima Jahnke ${\text {, } 2^{*}}^{{ }^{*}}$

\begin{abstract}
Background: The purpose of this study was to determine the ability of Blood Oxygen Level Dependent (BOLD) cardiovascular magnetic resonance (CMR) to detect stress-inducible myocardial ischemic reactions in the presence of angiographically significant coronary artery disease (CAD).

Methods: Forty-six patients (34 men; age $65 \pm 9$ years,) with suspected or known coronary artery disease underwent CMR at 3Tesla prior to clinically indicated invasive coronary angiography. BOLD CMR was performed in 3 short axis slices of the heart at rest and during adenosine stress $(140 \mu \mathrm{g} / \mathrm{kg} / \mathrm{min})$ followed by late gadolinium enhancement (LGE) imaging. In all 16 standard myocardial segments, $\mathrm{T2}^{*}$ values were derived at rest and under adenosine stress. Quantitative coronary angiography served as the standard of reference and defined normal myocardial segments (i.e. all 16 segments in patients without any CAD), ischemic segments (i.e. supplied by a coronary artery with $\geq 50 \%$ luminal narrowing) and non-ischemic segments (i.e. supplied by a non-significantly stenosed coronary artery in patients with significant CAD).
\end{abstract}

Results: Coronary angiography demonstrated significant CAD in 23 patients. BOLD CMR at rest revealed significantly lower T2* values for ischemic segments $(26.7 \pm 11.6 \mathrm{~ms})$ compared to normal $(31.9 \pm 11.9 \mathrm{~ms} ; \mathrm{p}<$ $0.0001)$ and non-ischemic segments (31.2 $\pm 12.2 \mathrm{~ms} ; \mathrm{p}=0.0003)$. Under adenosine stress $T 2^{*}$ values increased significantly in normal segments only (37.2 $\pm 14.7 \mathrm{~ms} ; \mathrm{p}<0.0001)$.

Conclusions: Rest and stress BOLD CMR at 3Tesla proved feasible and differentiated between ischemic, nonischemic, and normal myocardial segments in a clinical patient population. BOLD CMR during vasodilator stress identified patients with significant CAD.

\section{Background}

Cardiovascular magnetic resonance (CMR) is increasingly applied in clinical routine to determine myocardial perfusion [1-4] using contrast enhanced first-pass perfusion techniques [5-8]. Non-invasive characterization of myocardial microcirculation is thought to reflect myocardial tissue supply much better than mere luminographic detection and quantification of epicardial coronary stenosis, and has been shown to be useful for planning of revascularization procedures and cardiac risk stratification.

Blood Oxygen Level Dependent (BOLD) CMR is based on the paramagnetic properties of deoxyhemoglobin as an endogenous contrast agent with increased

\footnotetext{
* Correspondence: cjahnke@ukaachen.de

'Department of Internal Medicine/Cardiology, German Heart Institute, Berlin, Germany

Full list of author information is available at the end of the article
}

deoxyhemoglobin content leading to signal reduction on T2*- or T2-weighted images. Thus, BOLD CMR directly reflects myocardial oxygenation status $[9,10]$. Coronary artery stenosis leads to poststenotic microvascular dilatation in a compensatory effort to maintain sufficient myocardial oxygen supply [11] and blood-oxygen level dependent imaging has been successfully introduced to determine capillary reserve $[12,13]$.

However, data on myocardial BOLD CMR during vasodilator stress in patients with coronary artery disease (CAD) is limited to experimental studies [13-15] and small patient populations $[11,12]$. The main challenge was low signal intensity differences between normal and pathologic areas of myocardium at $1.5 \mathrm{~T}$, and therefore BOLD CMR at 3T may benefit from the inherently higher signal-to-noise ratio (SNR). Hence, in the present study we determined the ability of BOLD CMR to detect stress induced myocardial ischemia and to 
differentiate between ischemic, non-ischemic, and normal myocardial segments in a population of patients with suspected or known CAD.

\section{Materials and methods Study Group}

Forty-six consecutive patients (34 men; age $65 \pm 9$ years, range 40 to 81 years) referred for clinically indicated invasive coronary $\mathrm{x}$-ray angiography due to chest pain syndromes were prospectively enrolled. Patients were eligible if they had suspected or known CAD (with or without prior percutaneous revascularization or a history of previous myocardial infarction). Patients with prior coronary surgery or typical contraindications for CMR (e.g. incompatible metallic implants, claustrophobia) and administration of adenosine (asthma, AV-block $>$ grade I) were not considered.

All study participants were instructed to refrain from ß-blockers, antianginal medication, cigarettes, tea and coffee for at least 24 hours prior to CMR. Written informed consent was obtained from all subjects, and the Charité Institutional Review Board approved the study.

\section{CMR Imaging Protocol}

CMR was performed with the patient in the supine position using a 3T whole-body imager (Achieva 3T; Philips, Best, the Netherlands) equipped with a Quasar Dual gradient system $(40 \mathrm{mT} / \mathrm{m}$, slew rate $200 \mathrm{~T} / \mathrm{m} / \mathrm{sec})$. A six element cardiac synergy coil was used for signal reception and cardiac synchronization was done with the use of a Vector-ECG. All acquisitions were performed during short end-expiratory breath-holds. After acquisition of cine standard cardiac geometries for the assessment of left ventricular function a fast-gradientecho multi-echo sequence for BOLD CMR (3-slices of short axis geometry) was performed. Then, adenosine infusion $(140 \mu \mathrm{g} / \mathrm{kg} / \mathrm{min}$; maximal total infusion duration of 6 minutes) was started and the identical BOLD CMR sequence was repeated after at least 3 minutes of adenosine infusion. After termination of adenosine infusion, a bolus of $0.2 \mathrm{mmol} / \mathrm{kg}$ of gadolinium-DTPA was administered followed by late gadolinium enhancement (LGE) imaging 10 minutes later in identical short axis geometry with full left ventricular coverage.

\section{CMR Technique Cine Imaging}

Three short axis (apical, mid, and basal short axis views) and three long axis geometries (4-, 2-, and 3-chamber view) were acquired using an electrocardiogram-triggered balanced turbo field echo sequence (echo time $1.9 \mathrm{~ms}$, repetition time $4.0 \mathrm{~ms}$, flip angle $40^{\circ}$, spatial resolution $1.8 \times 1.8 \times 8 \mathrm{~mm}$ ) during repetitive endexpiratory breath-holding.

\section{BOLD CMR}

BOLD CMR imaging was carried out in the identical three short axis geometries of the left ventricle using an electrocardiogram-triggered, spoiled segmented gradient-echo sequence with six echo times (first echo at $2.7 \mathrm{~ms}$ with echo spacing of $1.7 \mathrm{~ms}$, repetition time $13 \mathrm{~ms}$, flip angle $35^{\circ}$, slice thickness $8 \mathrm{~mm}$, reconstructed spatial resolution $1.2 \times 1.2 \times 8 \mathrm{~mm}$, bandwidth $776 \mathrm{~Hz} /$ pixel). Image data acquisition was restricted to end-diastole in order to minimize cardiac motion related artefacts. A black blood dual inversion prepulse was used to nullify signal from flowing blood in the left ventricular cavity. Figure 1 shows a representative example of BOLD CMR at rest and during vasodilator stress.

\section{Late Gadolinium Enhancement Imaging}

Late gadolinium enhancement imaging was carried out in short axis orientation with full left ventricular coverage using a three-dimensional inversion prepared spoiled gradient echo sequence (echo time $1.8 \mathrm{~ms}$, repetition time $3.7 \mathrm{~ms}$, flip angle $15^{\circ}$; spatial resolution $1.5 \times$ $1.5 \times 5 \mathrm{~mm}$ ). The prepulse delay was determined from an inversion prepared cine scan (Look-Locker) and individually adjusted to optimally suppress signal from normal myocardium.

\section{Quantitative Coronary Angiography}

All patients underwent invasive coronary angiography in standard Judkins technique within 48 hours after CMR. The procedure was done according to angiographic guidelines using a simultaneous biplane, multidirectional and isocentric $\mathrm{x}$-ray system. At least two orthogonal views of every major coronary vessel and its side branches were acquired. Quantitative coronary angiography (Philips Inturis CardioView, QCA V3.3, Pie Medical Imaging) was performed off-line by an independent observer being unaware of the results of CMR. The severity of coronary stenosis was derived from one single view showing the maximal reduction in absolute luminal diameter and a significant coronary stenosis was defined as $\geq 50 \%$ luminal diameter reduction in vessels with $\geq 2 \mathrm{~mm}$ diameter; significant left main stenosis was considered double-vessel disease. In addition, myocardial segments were assigned to the supplying coronary artery based on a consensus read of the interventionalist and the CMR imager taking the respective coronary dominance type into account.

\section{Image analysis \\ BOLD CMR}

The BOLD CMR data sets were evaluated on a per segment basis according to the standardized 16-segment model [16]. For each segment, the time constant of the signal intensity decay over all echoes was derived. Moreover, the global $\mathrm{T} 2 *$ value per patient averaged over all segmental $\mathrm{T} 2 \%$ values was provided at rest and during 


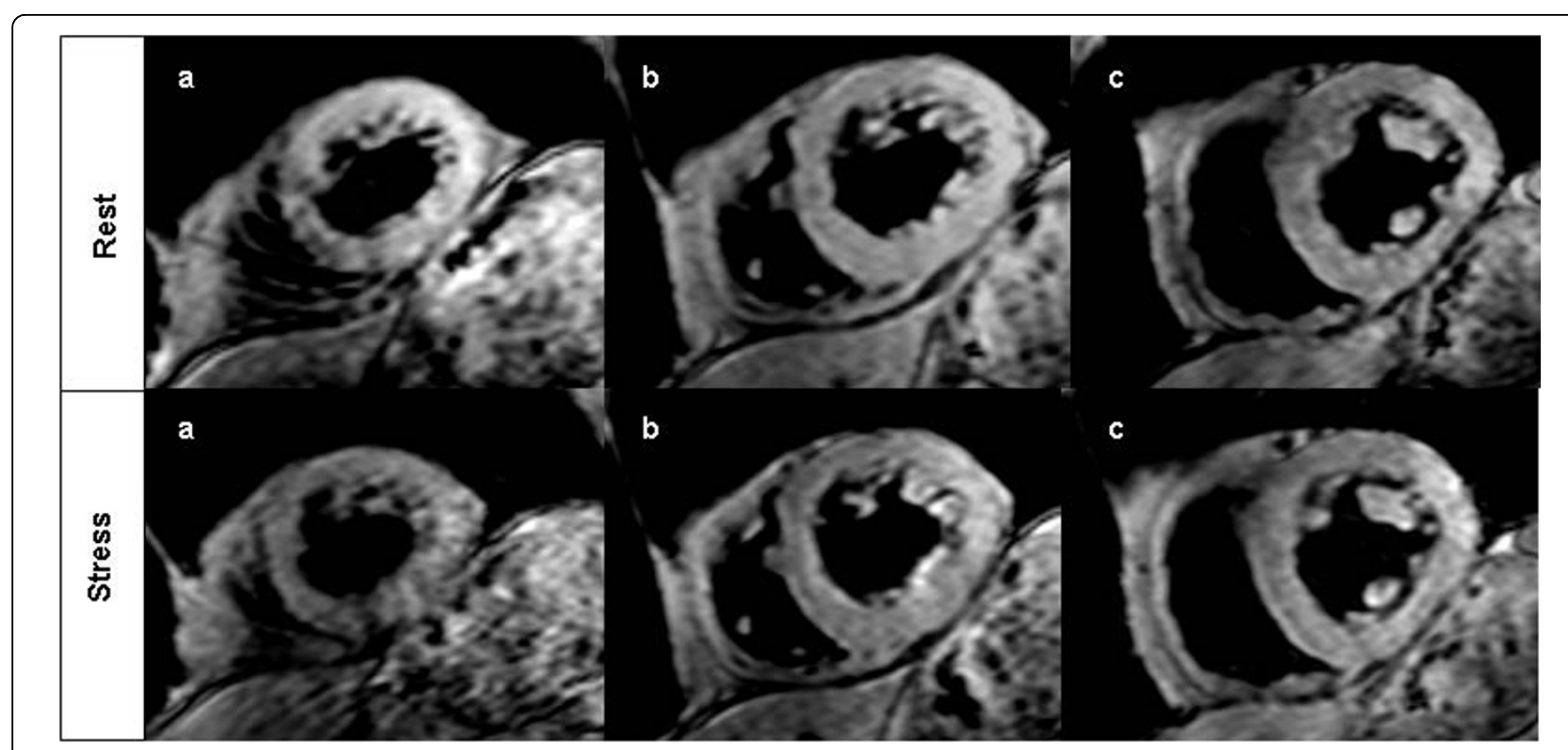

Figure 1 BOLD CMR at rest (upper row) and during adenosine stress (bottom row) showing the apical (a), midventricular (b) and basal slice (c).

stress. Image quality of T2* CMR measurements was graded visually on a per patient basis at rest and during stress on a four-point scale as excellent $(4=$ clear delineation of the left-ventricular myocardium with sharp endocardial contours, no motion artifacts), good (3 = clear delineation of the left-ventricular myocardium but mildly blurred endocardial contours, no motion artifacts), moderate $(2=$ moderately blurred endocardial contours, occurrence of respiratory/cardiac motion artifacts) or poor $(1=$ severely reduced delineation of leftventricular endocardial contours and severe motion artifacts). Occurrence of susceptibility artifacts caused by the heart-lung interface and cardiac veins was scored between 0 and $4(0=$ none, $1=$ minor, $2=$ moderate, $3=$ severe, $4=$ non-diagnostic).

\section{Late Gadolinium Enhancement Imaging}

LGE images were analyzed visually on a per segment basis regarding the presence of myocardial scar $\geq 25 \%$ transmurality with each myocardial segment being classified as normal or scarred.

\section{Statistical Analysis}

Statistical analysis was performed by using the SPSS software package release 17 (Chicago, Ill, USA). The paired Student's t test or Wilcoxon test and independent-samples $t$ test or Mann-Whitney test were used to test for differences within and between groups. All tests were two-tailed. The Kolmogorov-Smirnov test was used to test for normality. A P value of less than 0.05 was considered significant. For ROC analysis and the comparison of the area under the curve Medcalc ${ }^{\circ}$ release
9.2.1.0. (MedCalc Software, Belgium) was used. The areas under the curves were compared using the method of DeLong et al.[17]

\section{Results}

\section{Patients Characteristics and Hemodynamic Data}

A total of 46 consecutive patients were enrolled in this study. In 4 patients, CMR scans could not be completed: 2 patients were claustrophobic, and 2 did not tolerate adenosine. Hence, 42 (91\%) of 46 patients of this study cohort successfully completed the CMR examination and were included in the final analysis.Patient characteristics and hemodynamic data are summarized in table 1 and 2, respectively. Significant CAD was found in 23 patients $(54.8 \%)$ with ten patients (24\%) having singlevessel and 13 patients (31\%) multi-vessel disease. According to the results of QCA and LGE imaging a total of 672 myocardial segments were classified as follows: 272 (40.5\%) normal segments, 224 (33.3\%) nonischemic segments, 152 (22.6\%) ischemic segments. In addition, $24(3.6 \%)$ scarred segments were excluded from further analysis.

\section{Image Quality}

On BOLD CMR at rest, 15 patients showed excellent (35.7\%), 21 patients good (50.0\%), five patients moderate (11.9\%) and one patient poor image quality (2.4\%); under adenosine stress image quality was excellent in 11 patients $(26,2 \%)$, good in 21 patients $(50.0 \%)$, moderate in eight patients $(19.0 \%)$ and poor in two patients (4.8\%). The mean visual score of BOLD CMR was 


\section{Table 1 Patient Characteristics}

\begin{tabular}{ll}
\hline General parameters & \\
Sex, F/M (\%) & $12(29 \%) / 30(71 \%)$ \\
Age, $\mathrm{y}$ & $64 \pm 9$ \\
Range, y & $40-80$ \\
BMI, kg/m ${ }^{2}$ & $27.9 \pm 3.0$ \\
& \\
Historical information, $\mathbf{n}(\%)$ & \\
Hypertension & $36(86 \%)$ \\
Diabetes mellitus & $10(24 \%)$ \\
Hyperlipoproteinemia & $34(81 \%)$ \\
History of smoking & $7(17 \%)$ \\
CAD in family & $10(24 \%)$ \\
Suspected CAD & $30(71 \%)$ \\
Know CAD & $12(29 \%)$ \\
Previous PCl & $10(24 \%)$ \\
Previous myocardial infarction & $7(17 \%)$ \\
& \\
Medication, $\mathbf{n}$ (\%) & \\
ACE inhibitor & \\
Beta-blocker & $31(74 \%)$ \\
Calcium-channel blocker & $33(79 \%)$ \\
Statins & $8(19 \%)$ \\
Vessel disease, $\mathbf{n}$ (\%) & $35(83 \%)$ \\
Single & \\
Double & \\
Triple & $17(5 \%)$ \\
Multi & $10(12 \%)$ \\
Left anterior descending coronary artery & $17(40 \%)$ \\
Left circumflex coronary artery & $14(33 \%)$ \\
Right coronary artery & $10(24 \%)$ \\
\hline
\end{tabular}

$3.2 \pm 0.8$ at rest and $3.0 \pm 0.8$ under adenosine stress $(\mathrm{p}$ $=0.005$ ). Though all studies were of sufficient quality for analysis, segments demonstrating poor image quality were excluded from analysis (17 of 648, 2.6\%). The overall artifact score was significantly higher under adenosine stress than at rest $(1.6 \pm 0.6$ vs $1.4 \pm 0.5 ; \mathrm{p}=0.047$; for an imaging example see figure 2).

\section{BOLD CMR}

BOLD measurements at rest revealed significantly lower values for ischemic segments $(\mathrm{n}=148 ; 26.7 \pm 11.6 \mathrm{~ms})$ compared to normal segments $(\mathrm{n}=260 ; 31.9 \pm 11.9 \mathrm{~ms}$; $\mathrm{p}<0.0001)$ and non-ischemic segments $(\mathrm{n}=223 ; 31.2 \pm$ $12.2 \mathrm{~ms} ; \mathrm{p}=0.0003$ ). During adenosine stress $\mathrm{T}^{*}$ values demonstrated a significant increase in normal segments only $(37.2 \pm 14.7 \mathrm{~ms} ; \mathrm{p}<0.0001)$. On the contrary, $\mathrm{T} 2 *$ values of non-ischemic $(32.8 \pm 14.9 \mathrm{~ms} ; \mathrm{p}=0.19)$ and ischemic segments $(27.2 \pm 12.3 ; \mathrm{p}=0.06)$ did not
Table 2 Left Ventricular Function at rest and Hemodynamic Data

\begin{tabular}{ll}
\hline Left ventricular function & \\
LVEF, \% & $56 \pm 8$ \\
LVEDV, mL & $151 \pm 47$ \\
LVESV, mL & $71 \pm 30$ \\
& \\
Heart rate, bpm & $66 \pm 11$ \\
at rest & $83 \pm 15^{*}$ \\
max. Stress & \\
Systolic blood pressure, $\mathbf{m m H g}$ & $134 \pm 14$ \\
at rest & $132 \pm 17$ \\
max. Stress & \\
& \\
Heart rate-pressure product, bpm $\times \mathbf{~ m m H g}$ & $8874 \pm 1986$ \\
at rest & $11069 \pm 2653^{*}$ \\
max. Stress & \\
\hline${ }^{*}<0.01$ rest vs stress &
\end{tabular}

${ }^{*} p<0.01$ rest vs stress

significantly differ for the comparison of stress versus rest. When comparing stress $\mathrm{T} 2 *$ values of normal versus non-ischemic and ischemic segments significant differences were found $(\mathrm{p}=0.0007$ and $\mathrm{p}<0.0001$, respectively). Corresponding error bar charts are given in figure 3. Figure 4 shows the relationship between the changes of $\mathrm{T} 2 *$ values at rest and under adenosine stress and the degree of coronary artery stenosis.

The global T2* value of patients without significant CAD was $32.2 \pm 3.5 \mathrm{~ms}$ at rest and $37.4 \pm 6.3 \mathrm{~ms}$ under stress $(\mathrm{p}=0.0003)$. In patients with significant CAD the global T2* value was $30.3 \pm 5.3$ at rest and $30.7 \pm 5.8$ under stress $(\mathrm{p}=0.78)$. Imaging examples are provided in figure 5.

\section{Diagnostic Performance of BOLD CMR}

The area under the curve (AUC) of the ROC analysis (Figure 6) for the ability of T2* CMR imaging to detect significant $\mathrm{CAD}$ on a per patient basis differed significantly between rest $(0.61,95 \%$-CI $[0.44-0.75])$ and stress imaging $(0.82,95 \%$-CI [0.67-0.92], $\mathrm{p}=0.015)$. Using a cut-off value of $33.8 \mathrm{~ms}$, sensitivity and specificity for rest and stress were $78 \%$ and $21 \%$, and $78 \%$ and $68 \%$. AUC of the ROC analysis for the detection of patients with multivessel disease ( $\geq 2$ diseased vessels) under stress was 0.80 (95\% CI 0.63-0.92). Using a cut-off value $33.8 \mathrm{~ms}$, sensitivity and specificity were $77 \%$ and $68 \%$.

\section{Discussion}

In the present study, BOLD CMR at $3 \mathrm{~T}$ was shown to be feasible at $3 \mathrm{~T}$ resulting in an overall good image quality at rest and during vasodilator stress. BOLD 


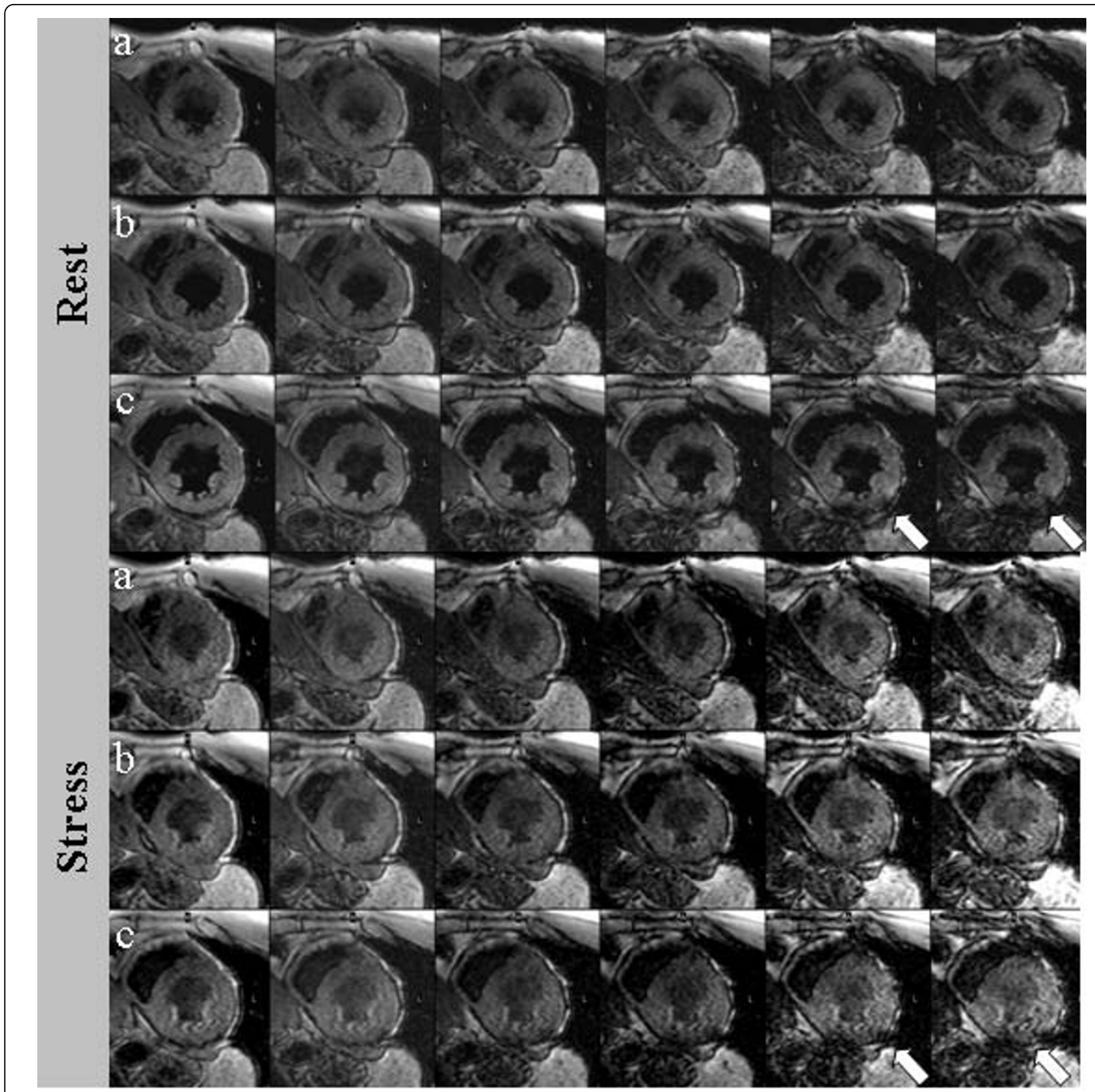

Figure 2 Example of rest (top rows) and stress (bottom rows) BOLD CMR showing the apical (a), mid-ventricular (b) and basal slice (c) with six echo times. Susceptibility artifacts (white arrow) occurred at long echo times predominantly in the inferolateral segment caused by the heart-lung interface.

CMR at rest and under stress conditions distinguished between normal, ischemic and non-ischemic myocardial segments. In addition, adenosine stress BOLD CMR gave a good diagnostic performance with regard to the detection of angiographically defined coronary stenosis.

The non-invasive detection of ischemic myocardium is one of the major strengths of CMR. BOLD imaging represents a promising alternative to first-pass CMR perfusion techniques, which rely on the application of exogenous contrast agents. BOLD imaging allows to directly assess the myocardial oxygenation status taking advantage of the paramagnetic properties of intravascular deoxyhemoglobin [11]. During adenosine stress, myocardial segments being supplied by normal coronary arteries show an increased oxygen content while deoxyhemoglobin concentration is low resulting in a higher signal on T2* or T2-weighted images. On the contrary, myocardial segments being supplied by a stenosed coronary artery exhibit a dilated capillary bed already under resting conditions in order to maintain sufficient blood 


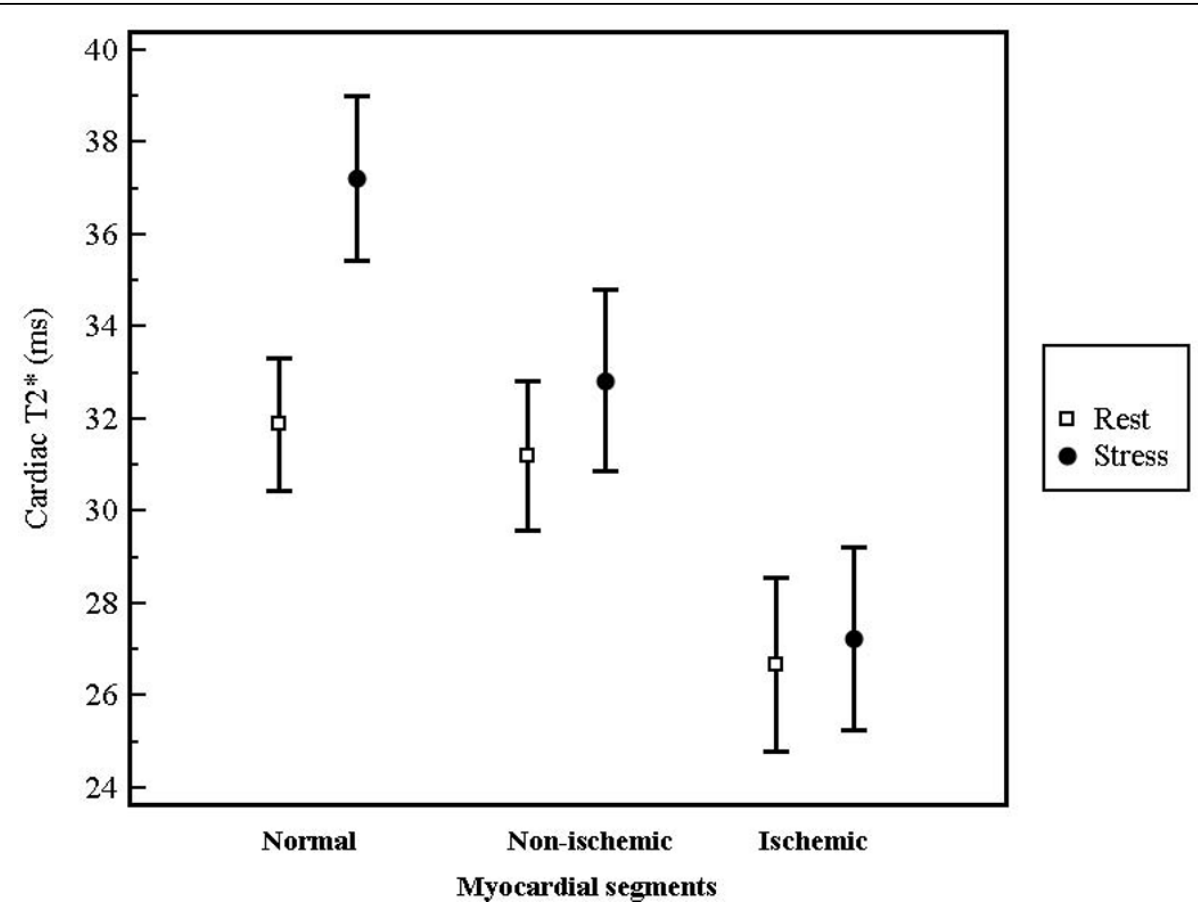

Figure 3 Error bar charts of $\mathrm{T}^{*}$ at rest and under adenosine stress comparing the mean values and corresponding $95 \%$ confidence intervals.

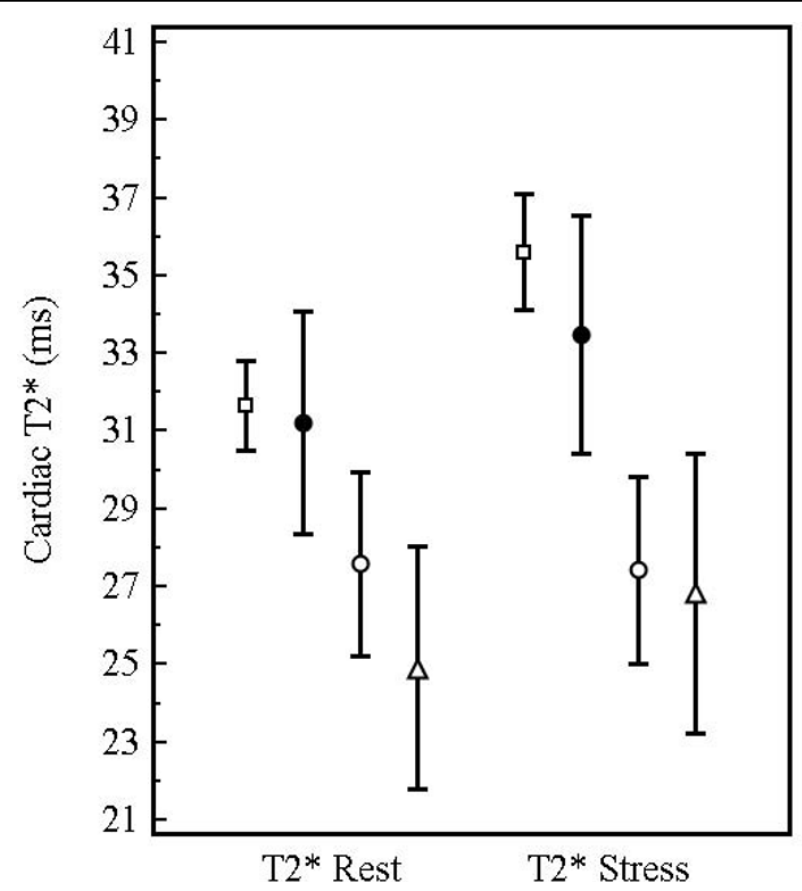

Degree of stenosis

ㅁ $0 \%$

- $1-49 \%$

○ $50-75 \%$

$\Delta 76-100 \%$

Figure 4 Relationship between T2* values (mean values and corresponding $95 \%$ confidence intervals) at rest and under adenosine stress and the degree of coronary artery stenosis. 


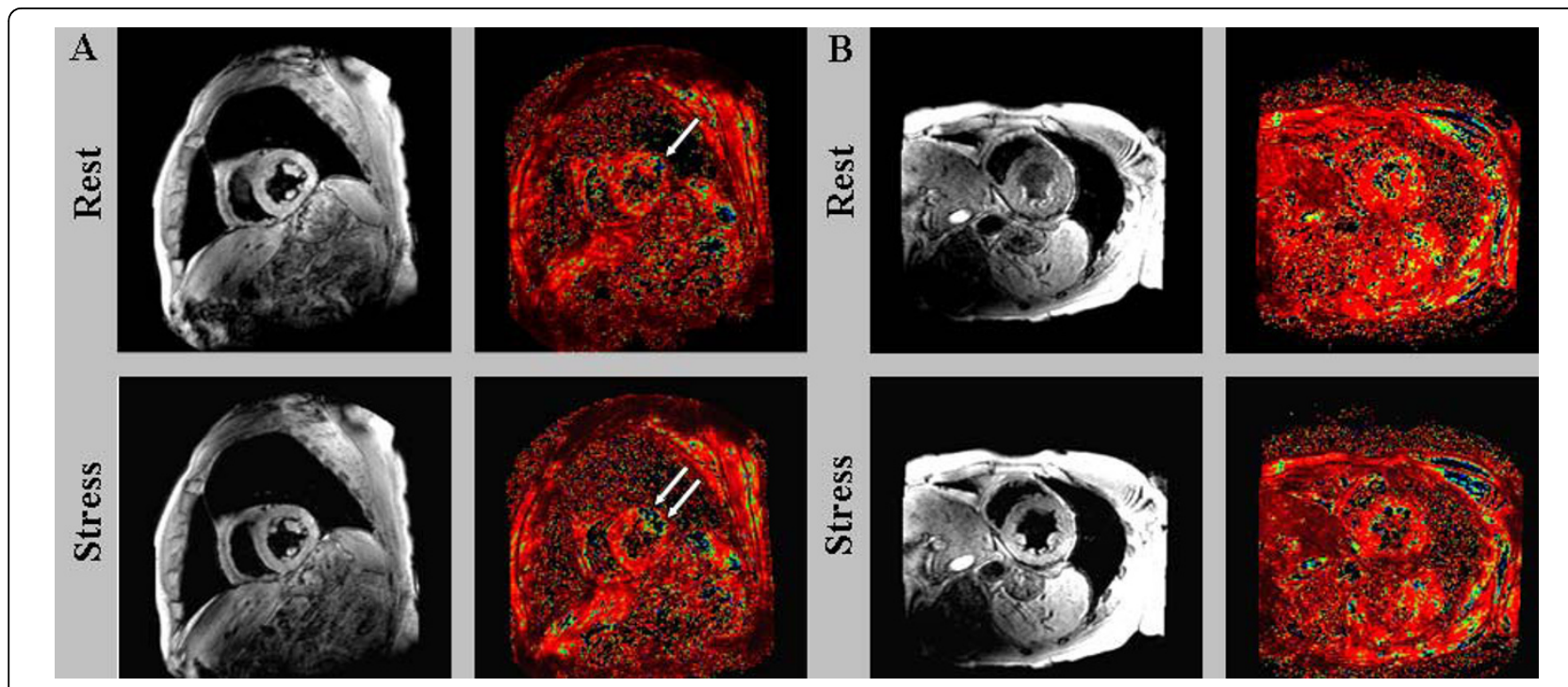

Figure 5 Imaging examples: Rest and stress BOLD CMR showing a patient with lateral ischemia (A) and a patient without coronary artery disease (B).

supply. Hence, additional vasodilatation under stress is minimal and the hyperaemic response is abolished.

In the present study, ischemic myocardial segments showed significantly reduced $\mathrm{T} 2 \%$ values under resting conditions, while during adenosine stress $\mathrm{T} 2 *$ values increased in normal myocardial segments only. Our findings were consistent with previous reports by
Wacker et al. and Niemi et al. [11,18], but somewhat contrasted the results by Friedrich et al. [12] even demonstrating a signal decrease during vasodilator stress in myocardial territories supplied by coronary arteries with $>75 \%$ luminal narrowing.

Interestingly, in the present study non-ischemic myocardial segments did not show a significant increase of

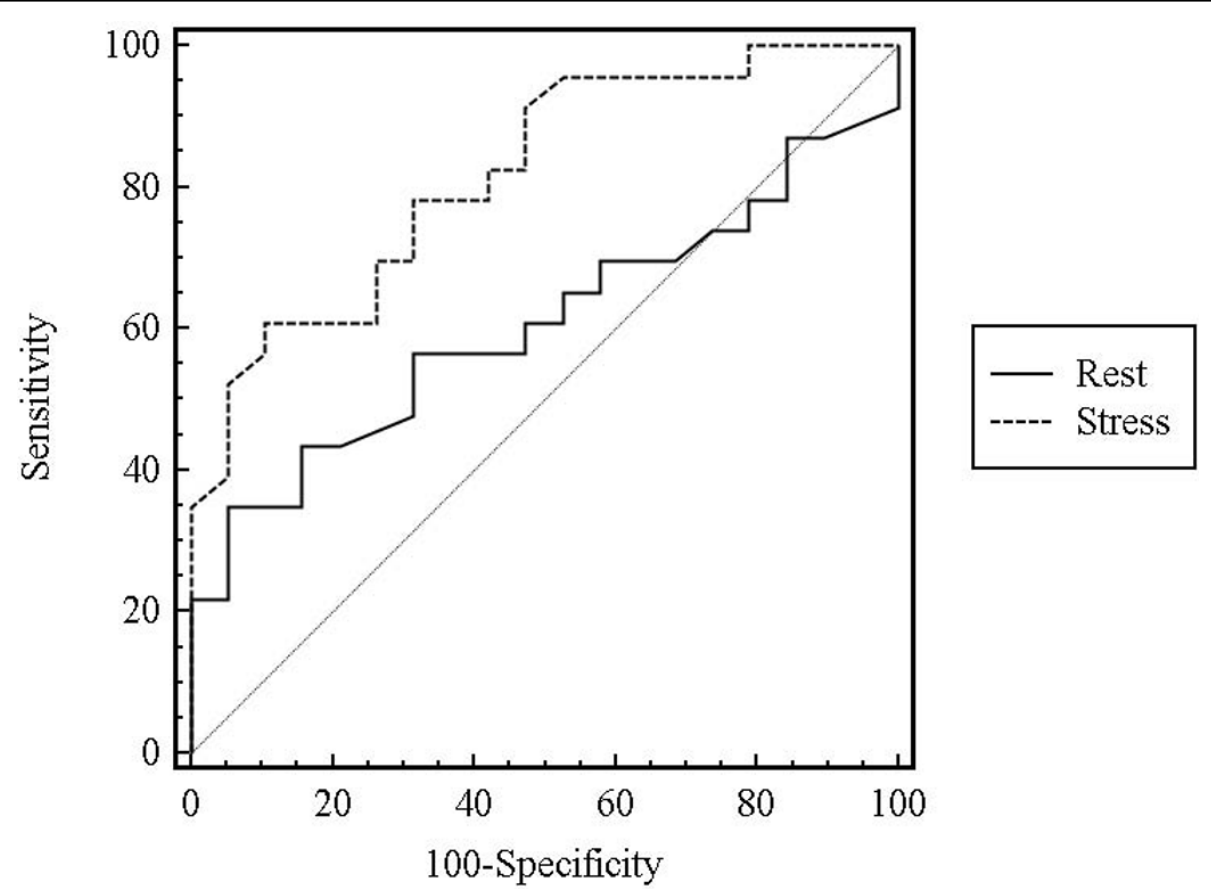

Figure 6 ROC analysis showing the diagnostic performance of rest and stress BOLD CMR to identify the presence of significant coronary artery disease. 
T2* values during stress. These findings may be explained by microvascular disease [19], which is present in myocardial segments of patients with CAD despite the absence of significant epicardial coronary artery narrowing. The T2* measurements of the current study showed a relation between the degree of coronary stenosis and the $\mathrm{T} 2 *$ value at rest and under adenosine stress.

The T2* CMR values at rest in patients without CAD were within the range of previously published data by Story et al. (33.3 $\pm 8.3 \mathrm{~ms}$, outliers were excluded) [20] and O'Regan et al. $(27.3 \pm 6.4 \mathrm{~ms})$ [21] using a singleslice image acquisition. However, in our study, the standard deviation of $\mathrm{T} 2$ * values was substantially larger, which can be explained by the imaging strategy employing a 3-slice acquisition per patient in order to achieve coverage of all 16-standard myocardial segments. Susceptibility artifacts appeared predominantly in the inferolateral segments of the myocardium on images with the longest echo times and resulted mainly from interference with cardiac veins [22] and the heart-lung interface [23]. In order to reduce these artifacts an endexpiratory breath hold was used as proposed by Wacker et al.[9] in conjunction with a black blood prepulse to reduce possible sources of artifacts arising from the blood pool. Furthermore, the use of only six echo times and a relatively short echo spacing and repletion time of $13 \mathrm{~ms}$ in our study prevents susceptibility artefacts caused by longer echo times [9]. Recently, O'Regan et al. [21] reported that black blood breath-hold multiecho $\mathrm{T} 2 *$ imaging can be adequately performed at $3 \mathrm{~T}$ and resulted in only minor susceptibility artifacts in the inferolateral region of the heart.

Importantly, the current study population consisted of consecutive patients with a high proportion of known CAD and prior myocardial infarctions but such patients were usually not considered in previous studies $[11,12,21]$. However, a direct comparison to first pass CMR perfusion or SPECT imaging would have been desirable and may be considered a limitation of the present study.

\section{Conclusions}

Rest and stress BOLD CMR at $3 \mathrm{~T}$ proved feasible and may be used to differentiate between ischemic, nonischemic, and normal myocardial segments in a clinical patient population. BOLD CMR during vasodilator stress identified patients with significant CAD.

\section{List of abbreviations}

CMR: Cardiovascular magnetic resonance; CAD: Coronary artery disease; LGE: Late gadolinium enhancement; SNR: Signal-to-noise ratio; QCA: Quantitative coronary angiography; ROC: Receiver under the operator curve; BMI: Body mass index; PCl: Percutaneous coronary intervention.

\section{Acknowledgements}

We are indebted to Uwe Kokartis for thoroughly performing the quantitative coronary angiographic measurements.

\section{Author details}

'Department of Internal Medicine/Cardiology, German Heart Institute, Berlin, Germany. ${ }^{2}$ Department of Cardiology, University Hospital RWTH Aachen, Germany. ${ }^{3}$ Philips Clinical Sciences, Hamburg, Germany.

\section{Authors' contributions}

RM: study design, data acquisition, image analysis, statistical analysis, manuscript drafting. IP: study design, image analysis, statistical analysis, manuscript drafting. BS: study design, literature research, manuscript drafting. EF: study design, manuscript drafting. RG: data acquisition, literature research. CJ: study design, statistical analysis, manuscript drafting, guarantator of integrity of entire study. All authors have made revisions to the manuscript and have read and approved the final version.

\section{Competing interests}

Bernhard Schnackenburg is an employee of Philips Medical Systems, Hamburg, Germany.

Received: 31 May 2010 Accepted: 22 September 2010

Published: 22 September 2010

\section{References}

1. Panting JR, Gatehouse PD, Yang GZ, Jerosch-Herold M, Wilke N, Firmin DN, Pennell DJ: Echo-planar magnetic resonance myocardial perfusion imaging: parametric map analysis and comparison with thallium SPECT. J Magn Reson Imaging 2001, 13:192-200.

2. Schwitter J, Nanz D, Kneifel S, Bertschinger K, Buchi M, Knusel PR, Marincek B, Luscher TF, von Schulthess GK: Assessment of myocardial perfusion in coronary artery disease by magnetic resonance: a comparison with positron emission tomography and coronary angiography. Circulation 2001, 103:2230-2235.

3. Schwitter J, Wacker CM, van Rossum AC, Lombardi M, Al-Saadi N, Ahlstrom H, Dill T, Larsson HB, Flamm SD, Marquardt M, Johansson L: MRIMPACT: comparison of perfusion-cardiac magnetic resonance with single-photon emission computed tomography for the detection of coronary artery disease in a multicentre, multivendor, randomized trial. Eur Heart J 2008, 29:480-489.

4. Kelle S, Graf K, Dreysse S, Schnackenburg B, Fleck E, Klein C: Evaluation of contrast wash-in and peak enhancement in adenosine first pass perfusion CMR in patients post bypass surgery. J Cardiovasc Magn Reson 2010, 12:28.

5. Al-Saadi N, Nagel E, Gross M, Bornstedt A, Schnackenburg B, Klein C, Klimek W, Oswald H, Fleck E: Noninvasive detection of myocardial ischemia from perfusion reserve based on cardiovascular magnetic resonance. Circulation 2000, 101:1379-1383.

6. Nagel E, Klein C, Paetsch I, Hettwer S, Schnackenburg B, Wegscheider K, Fleck E: Magnetic resonance perfusion measurements for the noninvasive detection of coronary artery disease. Circulation 2003, 108:432-437

7. Wilke $N$, Jerosch-Herold M, Wang Y, Huang Y, Christensen BV, Stillman AE, Ugurbil K, McDonald K, Wilson RF: Myocardial perfusion reserve: assessment with multisection, quantitative, first-pass MR imaging. Radiology 1997, 204:373-384.

8. Gebker R, Jahnke C, Paetsch I, Kelle S, Schnackenburg B, Fleck E, Nagel E: Diagnostic performance of myocardial perfusion MR at $3 \mathrm{~T}$ in patients with coronary artery disease. Radiology 2008, 247:57-63.

9. Wacker CM, Bock M, Hartlep AW, Beck G, van Kaick G, Ertl G, Bauer WR, Schad LR: Changes in myocardial oxygenation and perfusion under pharmacological stress with dipyridamole: assessment using T*2 and T1 measurements. Magn Reson Med 1999, 41:686-695.

10. Bauer WR, Nadler W, Bock M, Schad LR, Wacker C, Hartlep A, Ertl G: The relationship between the BOLD-induced $T(2)$ and $T(2)\left({ }^{*}\right)$ : a theoretical approach for the vasculature of myocardium. Magn Reson Med 1999, 42:1004-1010.

11. Wacker CM, Hartlep AW, Pfleger S, Schad LR, Ertl G, Bauer WR: Susceptibility-sensitive magnetic resonance imaging detects human 
myocardium supplied by a stenotic coronary artery without a contrast agent. J Am Coll Cardiol 2003, 41:834-840.

12. Friedrich MG, Niendorf T, Schulz-Menger J, Gross CM, Dietz R: Blood oxygen level-dependent magnetic resonance imaging in patients with stress-induced angina. Circulation 2003, 108:2219-2223.

13. Shea SM, Fieno DS, Schirf BE, Bi X, Huang J, Omary RA, Li D: T2-prepared steady-state free precession blood oxygen level-dependent MR imaging of myocardial perfusion in a dog stenosis model. Radiology 2005, 236:503-509.

14. Fieno DS, Shea SM, Li Y, Harris KR, Finn JP, Li D: Myocardial perfusion imaging based on the blood oxygen level-dependent effect using T2prepared steady-state free-precession magnetic resonance imaging. Circulation 2004, 110:1284-1290.

15. Zhang H, Gropler RJ, Li D, Zheng J: Assessment of myocardial oxygen extraction fraction and perfusion reserve with BOLD imaging in a canine model with coronary artery stenosis. J Magn Reson Imaging 2007, 26:72-79.

16. Cerqueira MD, Weissman NJ, Dilsizian V, Jacobs AK, Kaul S, Laskey WK Pennell DJ, Rumberger JA, Ryan T, Verani MS: Standardized myocardial segmentation and nomenclature for tomographic imaging of the heart: a statement for healthcare professionals from the Cardiac Imaging Committee of the Council on Clinical Cardiology of the American Heart Association. Circulation 2002, 105:539-542.

17. DeLong ER, DeLong DM, Clarke-Pearson DL: Comparing the areas under two or more correlated receiver operating characteristic curves: a nonparametric approach. Biometrics 1988, 44:837-845.

18. Niemi P, Poncelet BP, Kwong KK, Weisskoff RM, Rosen BR, Brady TJ, Kantor HL: Myocardial intensity changes associated with flow stimulation in blood oxygenation sensitive magnetic resonance imaging. Magn Reson Med 1996, 36:78-82.

19. Maseri A, Crea F, Kaski JC, Crake T: Mechanisms of angina pectoris in syndrome X. J Am Coll Cardiol 1991, 17:499-506.

20. Storey P, Thompson AA, Carqueville CL, Wood JC, de Freitas RA, Rigsby CK: R2* imaging of transfusional iron burden at $3 \mathrm{~T}$ and comparison with 1.5T. J Magn Reson Imaging 2007, 25:540-547.

21. O'Regan DP, Callaghan MF, Fitzpatrick J, Naoumova RP, Hajnal JV, Schmitz SA: Cardiac T2* and lipid measurement at 3.0 T-initial experience. Eur Radiol 2008, 18:800-805.

22. Reeder SB, Faranesh AZ, Boxerman JL, McVeigh ER: In vivo measurement of $T^{*} 2$ and field inhomogeneity maps in the human heart at $1.5 \mathrm{~T}$. Magn Reson Med 1998, 39:988-998.

23. Atalay MK, Poncelet BP, Kantor HL, Brady TJ, Weisskoff RM: Cardiac susceptibility artifacts arising from the heart-lung interface. Magn Reson Med 2001, 45:341-345.

doi:10.1186/1532-429X-12-54

Cite this article as: Manka et al: BOLD cardiovascular magnetic resonance at 3.0 tesla in myocardial ischemia. Journal of Cardiovascular Magnetic Resonance 2010 12:54

\section{Submit your next manuscript to BioMed Central and take full advantage of:}

- Convenient online submission

- Thorough peer review

- No space constraints or color figure charges

- Immediate publication on acceptance

- Inclusion in PubMed, CAS, Scopus and Google Scholar

- Research which is freely available for redistribution

Submit your manuscript at www.biomedcentral.com/submit
Biomed Central 